\title{
STRUCTURE FOR NONNEGATIVE SQUARE ROOTS OF UNBOUNDED NONNEGATIVE SELFADJOINT OPERATORS
}

\author{
BY
}

\author{
PENG-FEI YAO AND DE-XING FENG
}

Institute of Systems Science, Academia Sinica, Beijing, China

\begin{abstract}
It is well known that, for an unbounded nonnegative selfadjoint operator $A$ on a Hilbert space, there is a unique nonnegative square root $A^{1 / 2}$, which is frequently associated with the structural damping in many practical vibration systems. In this paper we develop a general theory for the structure of $A^{1 / 2}$, which includes the expression of $A^{1 / 2}$ and a program to find the domain of $A^{1 / 2}$ explicitly from the domain of $A$. The relationship between $A^{1 / 2}$ and related differential operators is determined for the selfadjoint differential operator $A$. Finally, the theoretical results given in this paper are applied to fourth-order "beam" operators and $n$-dimensional "wave" operators with sufficient complexity for applications to elastic vibration systems.
\end{abstract}

1. Introduction. Let $H$ be a Hilbert space with the inner product $(\cdot, \cdot)$ and the induced norm $\|\cdot\|$. Let $A$ be an unbounded nonnegative selfadjoint operator on $H$ and $\mathcal{L}$ a closed linear operator on $H$. In the last two decades, great attention has been focused on the following elastic system:

$$
\left\{\begin{array}{l}
\ddot{y}(t)+\mathcal{L} \dot{y}(t)+A y(t)=0 \\
y(0)=y_{0}, \dot{y}(0)=y_{1}
\end{array}\right.
$$

where a dot denotes $\frac{d}{d t}$, and $y, y_{0}, y_{1} \in H$. The usual procedure for dealing with the system (1.1) is as follows.

Letting $x_{1}=A^{1 / 2} y, x_{2}=y$, the system (1.1) can be transformed into an equivalent first-order evolution system

$$
\left\{\begin{array}{l}
\frac{d}{d t} \tilde{x}(t)=\mathcal{A} \tilde{x}(t) \\
\tilde{x}(0)=Y_{0}
\end{array}\right.
$$

where

$$
\tilde{x}(t)=\left(\begin{array}{l}
x_{1} \\
x_{2}
\end{array}\right), \quad \mathcal{A}=\left(\begin{array}{cc}
0 & A^{1 / 2} \\
-A^{1 / 2} & -\mathcal{L}
\end{array}\right), \quad Y_{0}=\left(\begin{array}{c}
A^{1 / 2} y_{0} \\
y_{1}
\end{array}\right)
$$

Received September 17, 1993.

1991 Mathematics Subject Classification. Primary 34B05, 47B06.

Key words and phrases. "elastic" systems, structural damping, Sobolev spaces.

This research was supported by the National Natural Science Foundation of China. 
and $A^{1 / 2}$ is the nonnegative square root of $A$. The domain of $\mathcal{A}$ is

$$
D\left(A^{1 / 2}\right) \times D\left(A^{1 / 2}\right)
$$

if $D(\mathcal{L}) \subset D\left(A^{1 / 2}\right)$, where $D(\mathcal{L})$ is the domain of $\mathcal{L}$. For many real systems, however, the structure of $D\left(A^{1 / 2}\right)$ is not clear except that $D(A)$ has special boundary conditions.

On the other hand, $A^{1 / 2} \dot{y}$ corresponds to structural damping for elastic vibration systems in [1] and is related to frequency proportional damping in [4]. Thus, it is necessary to understand the relationship between $A^{1 / 2}$ and differential operators in order that $A^{1 / 2} \dot{y}$ admits the proper physical interpretation if $A$ is a differential operator. D. L. Russell in [2] gave the relationship between $A^{1 / 2}$ and differential operators for fourth-order "beam" operators with the "symmetric" boundary conditions but the structure of $D\left(A^{1 / 2}\right)$ is not dealt with. A. V. Balakrishnan in [3], [4] obtained $D\left(A^{1 / 2}\right)$ and the relationship between $A^{1 / 2}$ and differential operators for two models of bending of uniform Bernoulli beams by the formula introduced in [5].

In this paper, we develop a general theory for the structure of the nonnegative square root of any unbounded nonnegative selfadjoint operator $A$. In Sec. 2, a program is given to find the domain of $A^{1 / 2}$ and the expression of $A^{1 / 2}$ is studied. Finally, in Secs. 3 and 4 , we apply the theoretical results developed in the paper to fourth-order elastic "beam" operators and $n$-dimensional "wave" operators, respectively.

2. The main results. Throughout this paper we make the following assumptions.

Let $H$ be a complex Hilbert space with the inner product $(\cdot, \cdot)$ and the induced norm $\|\cdot\|$, and let $A$ be an unbounded nonnegative selfadjoint operator on $H$ with compact resolvent and domain $D(A)$; let $A^{1 / 2}$ be the nonnegative square root of $A$. Note that $A$ is nonnegative if and only if

$$
(A x, x) \geq 0, \quad \forall x \in D(A)
$$

The operator $A$ is said to be positive if

$$
(A x, x)>0, \quad \forall x \in D(A), x \neq 0 .
$$

Let $H_{1}$ be another complex Hilbert space with the inner product $(\cdot, \cdot)_{1}$ and the induced norm $\|\cdot\|_{1}$ and $B: D(B) \subset H \rightarrow H_{1}$ be a closed linear operator such that

$$
\begin{gathered}
D(B) \supset D(A) ; \\
(A x, x) \geq\|B x\|_{1}^{2}, \quad \forall x \in D(A) .
\end{gathered}
$$

Set

$$
\begin{aligned}
{[x, y]=} & (x, A y)-(B x, B y)_{1}, \quad \forall x \in D(B), \forall y \in D(A), \\
& M_{0}=\left\{x \mid x \in D(A),(x, A x)=\|B x\|_{1}^{2}\right\} .
\end{aligned}
$$

We introduce several definitions in preparation for development of the structure of $A^{1 / 2}$. It should be noted that, throughout this paper, the definitions, given specially, all belong to the authors. 
Definition 2.1. $\left(B, H_{1}\right)$ is said to be a pseudo-square-root of $A$ if $\bar{M}_{0}=H$, where $\bar{M}_{0}$ is the closure of $M_{0}$ in $H$.

REMARK 2.1. Any nonnegative selfadjoint operator $A$ has at least one pseudo-squareroot. For example, $\left(A^{1 / 2}, H\right)$ is a pseudo-square-root of $A$, where $B=A^{1 / 2}$ and $H_{1}=H$.

EXAMPLE 2.1. Let $\Omega$ be a bounded domain of $R^{n}$ with smooth boundary $\partial \Omega$ of $C^{2}$ and $H=L^{2}(\Omega)$. Consider the following Laplace operator:

$$
\begin{gathered}
D(A)=\left\{u\left|u \in H^{2}(\Omega),\left(\frac{\partial u}{\partial n}+\alpha u\right)\right|_{\partial \Omega}=0\right\}, \\
A u=-\Delta u,
\end{gathered}
$$

where $\alpha \geq 0, \frac{\partial}{\partial n}$ is the normal derivative, and $\Delta u=\sum_{j=1}^{n} \frac{\partial^{2} u}{\partial x_{j}^{2}}$. It is well known that $A$ is an unbounded nonnegative selfadjoint operator on $L^{2}(\Omega)$.

Set $H_{1}=\left(L^{2}(\Omega)\right)^{n} ; H_{1}$ is a product Hilbert space with the inner product

$$
\langle\tilde{u}, \tilde{v}\rangle=\sum_{j=1}^{n}\left(u_{j}, v_{j}\right)_{L^{2}(\Omega)}, \quad \forall \tilde{u}=\left(u_{1}, u_{2}, \ldots, u_{n}\right)^{\tau}, \tilde{v}=\left(v_{1}, v_{2}, \ldots, v_{n}\right)^{\tau} \in\left(L^{2}(\Omega)\right)^{n} .
$$

Define $B: L^{2}(\Omega) \rightarrow\left(L^{2}(\Omega)\right)^{n}$ by

$$
D(B)=H^{1}(\Omega), \quad B u=\nabla u=\left(\frac{\partial u}{\partial x_{1}}, \ldots, \frac{\partial u}{\partial x_{n}}\right)^{\tau} .
$$

Conditions (2.1) and (2.2) can easily be verified by using Green's formula, and

$$
H_{0}(\Omega)=\left\{u\left|u \in H^{2}(\Omega), u\right|_{\partial \Omega}=\left.\frac{\partial u}{\partial n}\right|_{\partial \Omega}=0\right\} \subset M_{0} .
$$

Hence $\left(\nabla,\left(L^{2}(\Omega)\right)^{n}\right)$ is a pseudo-square-root of $A$.

Definition 2.2. Let $\left(B, H_{1}\right)$ be a pseudo-square-root of $A$. Let $H_{\partial}^{+}$be a Hilbert space with the inner product $(\cdot, \cdot)_{\partial}^{+}$and the induced norm $\|\cdot\|_{\partial}^{+}$. If there exist a bounded positive selfadjoint operator $\Upsilon: H_{\partial}^{+} \rightarrow H_{\partial}^{+}$and a mapping $\Gamma: D(B) \rightarrow H_{\partial}^{+}$such that

$$
[x, y]=(\Upsilon \Gamma x, \Gamma y)_{\partial}^{+}, \quad \forall x, y \in D(A),
$$

then we say that $\left(H_{\partial}^{+}, \Upsilon, \Gamma\right)$ is a positive boundary space of $A$ corresponding to $B$.

ExAmple 2.2. Let $H, A, H_{1}$, and $B$ be given by Example 2.1. By Green's formula, we have

$$
\begin{aligned}
{[u, v] } & =-\int_{\partial \Omega} u \frac{\partial \bar{v}}{\partial n} d \sigma \\
& =\alpha \int_{\partial \Omega} u \bar{v} d \sigma, \quad \forall u \in H^{1}(\Omega), \forall v \in D(A) .
\end{aligned}
$$

Set $H_{\partial}^{+}=L^{2}(\partial \Omega)$ and $\Upsilon=\alpha I$, where $I$ is the identity mapping on $L^{2}(\partial \Omega)$. Define $\Gamma: H^{1}(\Omega) \rightarrow L^{2}(\partial \Omega)$ by

$$
\Gamma u=\left.u\right|_{\partial \Omega}, \quad \forall u \in H^{1}(\Omega) .
$$

It is easy to verify from $(2.4)$ that $\left(L^{2}(\partial \Omega), \alpha I, \Gamma\right)$ is a positive boundary space of $A$ corresponding to $B$.

The following proposition follows from a direct check. 
Proposition 2.1. Let $\left(B, H_{1}\right)$ be a pseudo-square-root of $A$ and $\left(H_{\partial}^{+}, \Upsilon, \Gamma\right)$ a positive boundary space of $A$ corresponding to $B$. Then

$$
\begin{gathered}
|[x, y]| \leq 2\left\|A^{1 / 2} x\right\|\left\|A^{1 / 2} x\right\|\left\|A^{1 / 2} y\right\|, \quad \forall x, y \in D\left(A^{1 / 2}\right) ; \\
\left|(\Upsilon \Gamma x, \Gamma y)_{\partial}^{+}\right| \leq 2\left\|A^{1 / 2} x\right\|\left\|A^{1 / 2} y\right\|, \quad \forall x, y \in D(A) .
\end{gathered}
$$

It is well known that the spectrum of $A$ consists of eigenvalues:

$$
0 \leq \lambda_{1} \leq \lambda_{2} \leq \cdots \leq \lambda_{n} \leq \lambda_{n+1} \leq \cdots
$$

with $\lim _{n \rightarrow \infty} \lambda_{n}=+\infty$, where the multiple eigenvalues are listed according to their algebraic multiplicities. There exists an orthonormal basis $\left\{x_{n}\right\}$ of $H$ such that

$$
A x_{n}=\lambda_{n} x_{n}, \quad n=1,2, \ldots
$$

Let $k$ be the nonnegative integer such that

$$
\lambda_{j}=0 \quad \text { if } j \leq k ; \quad \lambda_{j}>0 \quad \text { if } j \geq k+1 .
$$

If $A$ is positive, then $k=0$.

Proposition 2.2. Let $\left(B, H_{1}\right)$ be a pseudo-square-root of $A$ and $\left(H_{\partial}^{+}, \Upsilon, \Gamma\right)$ a positive boundary space of $A$ corresponding to $B$. Set

$$
g_{n}=\frac{1}{\sqrt{\lambda_{n}}} \Gamma x_{n}, \quad \psi_{n}=\frac{1}{\sqrt{\lambda_{n}}} B x_{n}, \quad n=k+1, k+2, \ldots
$$

Then

$$
\left\{\left(\begin{array}{c}
\Upsilon^{1 / 2} g_{n} \\
\psi_{n}
\end{array}\right) \mid n \geq k+1\right\}
$$

is an orthonormal set in the product Hilbert space $H_{\partial}^{+} \times H_{1}$, where the inner product of $H_{\partial}^{+} \times H_{1}$ is defined by

$$
\left\langle\left(\begin{array}{c}
f \\
\psi
\end{array}\right),\left(\begin{array}{c}
g \\
\phi
\end{array}\right)\right\rangle=(f, g)+(\psi, \phi)_{1}, \quad \forall f, g \in H_{\partial}^{+}, \forall \psi, \phi \in H_{1} .
$$

Proof. For any $n, m \geq k+1$, we have

$$
\begin{aligned}
\left(x_{n}, x_{m}\right) & =\frac{1}{\lambda_{m}}\left(x_{n}, A x_{m}\right)=\frac{1}{\lambda_{m}}\left[x_{n}, x_{m}\right]+\frac{1}{\lambda_{m}}\left(B x_{n}, B x_{m}\right)_{1} \\
& =\sqrt{\frac{\lambda_{n}}{\lambda_{m}}}\left[\left(\Upsilon g_{n}, g_{m}\right)_{\partial}^{+}+\left(\psi_{n}, \psi_{m}\right)_{1}\right] \\
& =\sqrt{\frac{\lambda_{n}}{\lambda_{m}}}\left\langle\left(\begin{array}{c}
\Upsilon^{1 / 2} g_{n} \\
\psi_{n}
\end{array}\right),\left(\begin{array}{c}
\Upsilon^{1 / 2} g_{m} \\
\psi_{m}
\end{array}\right)\right\rangle .
\end{aligned}
$$

The orthonormality of $\left\{x_{n}\right\}$ in $H$ yields the orthonormality of

$$
\left\{\left(\begin{array}{c}
\Upsilon^{1 / 2} g_{n} \\
\psi_{n}
\end{array}\right) \mid n \geq k+1\right\}
$$

in $H_{\partial}^{+} \times H_{1}$.

First, we consider the structure of $D\left(A^{1 / 2}\right)$. 
Theorem 2.1. Let $\left(B, H_{1}\right)$ be a pseudo-square-root of $A$ and $\left(H_{\partial}^{+}, \Upsilon, \Gamma\right)$ a positive boundary space of $A$ corresponding to $B$. Then

$$
D\left(A^{1 / 2}\right)=\left\{x \mid x \in D(B),[x, y]=(\Upsilon \Gamma x, \Gamma y)_{\partial}^{+}, \forall y \in D(A)\right\} .
$$

Proof. Let $x \in D\left(A^{1 / 2}\right)$. Then there are $\left\{z_{n}\right\}$ in $D(A)$ such that in $H$

$$
z_{n} \rightarrow x \quad \text { and } \quad A^{1 / 2} z_{n} \rightarrow A^{1 / 2} x, \quad \text { as } n \rightarrow \infty
$$

By Proposition 2.1 and (2.2), we have that $x \in D(B)$ and that

$$
\begin{aligned}
{[x, y] } & =\lim _{n \rightarrow \infty}\left[z_{n}, y\right]=\lim _{n \rightarrow \infty}\left(\Upsilon \Gamma z_{n}, \Gamma y\right)_{\partial}^{+} \\
& =(\Upsilon \Gamma x, y)_{\partial}^{+}, \quad \forall y \in D(A) .
\end{aligned}
$$

Conversely, suppose $x \in D(B)$ such that

$$
[x, y]=(\Upsilon \Gamma x, y)_{\partial}^{+}, \quad \forall y \in D(A) .
$$

Set

$$
x=\sum_{j=1}^{\infty} \alpha_{n} x_{n}
$$

Then

$$
\begin{aligned}
\alpha_{n} & =\left(x, x_{n}\right)=\frac{1}{\lambda_{n}}\left(x, A x_{n}\right) \\
& =\frac{1}{\lambda_{n}}\left[x, x_{n}\right]+\frac{1}{\lambda_{n}}\left(B x, B x_{n}\right)_{1} \\
& =\frac{1}{\sqrt{\lambda_{n}}}\left[x, \frac{1}{\sqrt{\lambda_{n}}} x_{n}\right]+\frac{1}{\sqrt{\lambda_{n}}}\left(B x, \psi_{n}\right)_{1}, \quad \forall n \geq k+1,
\end{aligned}
$$

or equivalently

$$
\begin{aligned}
\sqrt{\lambda_{n}} \alpha_{n} & =\left[x, \frac{1}{\sqrt{\lambda_{n}}} x_{n}\right]+\left(B x, \psi_{n}\right)_{1} \\
& =\left(\Upsilon^{1 / 2} \Gamma x, \Upsilon^{1 / 2} g_{n}\right)_{\partial}^{+}+\left(B x, \psi_{n}\right)_{1} \\
& =\left\langle\left(\begin{array}{c}
\Upsilon^{1 / 2} \Gamma x \\
B x
\end{array}\right),\left(\begin{array}{c}
\Upsilon^{1 / 2} g_{n} \\
\psi_{n}
\end{array}\right)\right\rangle, \quad \forall n \geq k+1
\end{aligned}
$$

Thus Proposition 2.2 yields

$$
\sum_{n=k+1}^{\infty}\left|\alpha_{n}\right|^{2} \lambda_{n} \leq\left\|\left(\begin{array}{c}
\Upsilon^{1 / 2} \Gamma x \\
B x
\end{array}\right)\right\|_{H_{\partial}^{+} \times H_{1}}^{2}<+\infty
$$

so that $x \in D\left(A^{1 / 2}\right)$.

When $M_{0}=D(A)$, we can choose $H_{\partial}^{+}=\{0\}$. Thus we have 
Corollary 2.1. Let $\left(B, H_{1}\right)$ be a pseudo-square-root of $A$. If $M_{0}=D(A)$, then

$$
D\left(A^{1 / 2}\right)=\left\{x \mid x \in D(B),[x, y]=0, \forall y \in M_{0}\right\} .
$$

EXAmple 2.3. Let $A$ be a "string vibration" operator on $L^{2}(0,1)$, i.e., $A u=-u^{\prime \prime}(\cdot)$, $u \in D(A)$, such that

$$
H_{0}^{2}(0,1) \subset D(A) .
$$

Set $H_{1}=L^{2}(0,1)$. The closed linear operator $B: L^{2}(0,1) \rightarrow L^{2}(0,1)$ is defined by

$$
D(B)=H^{1}(0,1), \quad B u=i D u=i u^{\prime}(\cdot) .
$$

By (2.10) and integration by parts it is easily checked that $\left(i D, L^{2}(0,1)\right)$ is a pseudosquare-root of $A$.

(i) If $A$ has the following boundary conditions,

$$
D(A)=\left\{u \mid u \in H^{2}(0,1), u(0)=u(1)=0\right\},
$$

it is easily verified that $M_{0}=D(A)$. For any $u \in H^{1}(0,1)$, it is clear that $u$ satisfies

$$
[u, v]=u(0) \overline{v^{\prime}(0)}-u(1) \overline{v^{\prime}(1)}=0, \quad \forall v \in D(A)
$$

if and only if $u(0)=u(1)=0$. By Corollary 2.1, we have

$$
D\left(A^{1 / 2}\right)=\left\{u \mid u \in H^{1}(0,1), u(0)=u(1)=0\right\}=H_{0}^{1}(0,1) .
$$

(ii) If $A$ has the boundary conditions

$$
D(A)=\left\{u \mid u \in H^{2}(0,1), u^{\prime}(0)=u^{\prime}(1)=0\right\},
$$

then $M_{0}=D(A)$. For any $u \in H^{1}(0,1)$, it is clear that

$$
[u, v]=u(0) \overline{v^{\prime}(0)}-u(1) \overline{v^{\prime}(1)}=0, \quad \forall v \in D(A) .
$$

Therefore, Corollary 2.1 yields

$$
D\left(A^{1 / 2}\right)=H^{1}(0,1) .
$$

(iii) If $A$ has the boundary conditions

$$
D(A)=\left\{u \mid u \in H^{2}(0,1), u(0)=u^{\prime}(1)=0\right\},
$$

by a similar process, we can obtain

$$
D\left(A^{1 / 2}\right)=\left\{u \mid u \in H^{1}(0,1), u(0)=0\right\} .
$$

(iv) For the boundary conditions

$$
D(A)=\left\{u \mid u \in H^{2}(0,1), u(0)=u^{\prime}(0), u(1)=0\right\},
$$

set $H_{\partial}^{+}=\mathbb{C}$ and $\Upsilon=1$. Define $\Gamma: H^{1}(0,1) \rightarrow C$ by $\Gamma u=u(0)$. Then $(C, 1, \Gamma)$ is a positive boundary space of $A$ corresponding to $i D$. Since

$$
\begin{aligned}
{[u, v] } & =-\left.u(x) \overline{v^{\prime}(x)}\right|_{0} ^{1}=u(0) \overline{v^{\prime}(0)}-u(1) \overline{v^{\prime}(1)} \\
& =(\Upsilon \Gamma u, \Gamma v)_{\partial}^{+}-u(1) \overline{v^{\prime}(1)}, \quad \forall u \in H^{1}(0,1), \forall v \in D(A),
\end{aligned}
$$

we have from Theorem 1.1 that

$$
D\left(A^{1 / 2}\right)=\left\{u \mid u \in H^{1}(0,1), u(1)=0\right\} .
$$

Now we consider explicit representations of $A^{1 / 2}$. 
Proposition 2.3. Let $\left(B, H_{1}\right)$ be a pseudo-square-root of $A$ and $\left(H_{\partial}^{+}, \Upsilon, \Gamma\right)$ a positive boundary space of $A$ corresponding to $B$. Let

$$
\mathcal{M}_{0}=\overline{\operatorname{span}}\left\{\left(\begin{array}{c}
\Upsilon^{1 / 2} g_{n} \\
\psi_{n}
\end{array}\right) \mid n \geq k+1\right\}
$$

be a closed linear subspace of $H_{\partial}^{+} \times H_{1}$. Then

$$
\mathcal{M}_{0}=\left\{\left(\begin{array}{c}
\Upsilon^{1 / 2} \Gamma x \\
B x
\end{array}\right) \mid x \in D\left(A^{1 / 2}\right)\right\}
$$

Proof. By Theorem 2.1 and (2.2), we have

$$
\begin{aligned}
\left(\left\|\Upsilon^{1 / 2} \Gamma x\right\|_{\partial}^{+}\right)^{2} & =[x, x]=(x, A x)-(B x, B x)_{1} \\
& \leq 2\left\|A^{1 / 2} x\right\|^{2}, \quad \forall x \in D\left(A^{1 / 2}\right)
\end{aligned}
$$

We have that $A^{1 / 2} x_{n}=0, \forall n, 1 \leq n \leq k$, since $A x_{n}=0$, for any $1 \leq n \leq k$. Therefore, (2.2) yields

$$
B x_{n}=0, \quad \forall n, 1 \leq n \leq k .
$$

By (2.6), it follows that

$$
\Gamma x_{n}=0, \quad \forall n, 1 \leq n \leq k .
$$

Suppose $x \in D\left(A^{1 / 2}\right)$. Set $x=\sum_{n=1}^{\infty} \alpha_{n} x_{n}$. From (2.6) together with (2.7), we have

$$
\Upsilon^{1 / 2} \Gamma x=\sum_{n=1}^{\infty} \alpha_{n} \Upsilon^{1 / 2} \Gamma x_{n}=\sum_{n=k+1}^{\infty} \alpha_{n} \Upsilon^{1 / 2} \Gamma x_{n}=\sum_{n=k+1}^{\infty} \sqrt{\lambda_{n}} \alpha_{n} \Upsilon^{1 / 2} g_{n}
$$

By (2.2) and (2.5), it follows that

$$
B x=\sum_{n=1}^{\infty} \alpha_{n} B x_{n}=\sum_{n=k+1}^{\infty} \alpha_{n} B x_{n}=\sum_{n=k+1}^{\infty} \sqrt{\lambda_{n}} \alpha_{n} \psi_{n}
$$

Therefore, (2.18) and (2.19) mean that

$$
\left(\begin{array}{c}
\Upsilon^{1 / 2} \Gamma x \\
B x
\end{array}\right)=\sum_{n=k+1}^{\infty} \sqrt{\lambda_{n}} \alpha_{n}\left(\begin{array}{c}
\Upsilon^{1 / 2} g_{n} \\
\psi_{n}
\end{array}\right) \in \mathcal{M}_{0}, \quad \forall x=\sum_{n=1}^{\infty} \alpha_{n} x_{n} \in D\left(A^{1 / 2}\right)
$$

Conversely, suppose $(g, \psi)^{\tau} \in \mathcal{M}_{0}$, where $g \in H_{\partial}^{+}$and $\psi \in H_{1}$. By Proposition 2.2, we have

$$
\left(\begin{array}{c}
g \\
\psi
\end{array}\right)=\sum_{n=k+1}^{\infty} \beta_{n}\left(\begin{array}{c}
\Upsilon^{1 / 2} g_{n} \\
\psi_{n}
\end{array}\right)
$$

where $\sum_{n=k+1}^{\infty}\left|\beta_{n}\right|^{2}<+\infty$. Setting $x=\sum_{n=k+1}^{\infty} \frac{\beta_{n}}{\sqrt{\lambda_{n}}} x_{n}$, it is easily checked that $x \in D\left(A^{1 / 2}\right)$ and $(g, \psi)^{\tau}=\left(\Upsilon^{1 / 2} \Gamma x, B x\right)^{\tau}$. 
Proposition 2.4. Let $\left(B, H_{1}\right)$ be a pseudo-square-root of $A$ and $\left(H_{\partial}^{+}, \Upsilon, \Gamma\right)$ a positive boundary space of $A$ corresponding to $B$. Define $\mathcal{B}: H \rightarrow H_{\partial}^{+} \times H_{1}$ by

$$
D(\mathcal{B})=D\left(A^{1 / 2}\right), \quad \mathcal{B} x=\left(\begin{array}{c}
\Upsilon^{1 / 2} \Gamma x \\
B x
\end{array}\right)
$$

Then

$$
\mathcal{B}: H \rightarrow H_{\partial}^{+} \times H_{1}
$$

is a closed linear operator.

Proof. Suppose that $\left\{z_{n}\right\} \subset D\left(A^{1 / 2}\right)$ such that in $H, z_{n} \rightarrow x$, as $n \rightarrow \infty$, such that in $H_{\partial}^{+}, \Upsilon^{1 / 2} \Gamma z_{n} \rightarrow g$, as $n \rightarrow \infty$, and such that in $H_{1}, B z_{n} \rightarrow \psi$, as $n \rightarrow \infty$, where $x \in H$, $g \in H_{\partial}^{+}$, and $\psi \in H_{1}$. From the closedness of $B$ it follows that $x \in D(B)$ and $B x=\psi$. From (2.6) and (2.2), it follows that $\Upsilon^{1 / 2} \Gamma x=g$. Therefore $\mathcal{B}$ is closed.

Now we consider the expression of $A^{1 / 2}$.

ThEOREM 2.2. Let $\left(B, H_{1}\right)$ be a pseudo-square-root of $A$ and $\left(H_{\partial}^{+}, \Upsilon, \Gamma\right)$ a positive boundary space of $A$ corresponding to $B$. Then there exists a bounded linear operator $\mathcal{Q}: H_{\partial}^{+} \times H_{1} \rightarrow H$ such that

(i) $\|\mathcal{Q}\| \leq 1, \mathcal{Q}: \mathcal{M}_{0} \rightarrow H$ is isometric;

(ii) $A^{1 / 2} x=\mathcal{Q B} x$, for any $x \in D\left(A^{1 / 2}\right)$.

Proof. For any $(g, \psi)^{\tau} \in \mathcal{M}_{0}$, set

$$
\left(\begin{array}{c}
g \\
\psi
\end{array}\right)=\sum_{n=k+1}^{\infty} \alpha_{n}\left(\begin{array}{c}
\Upsilon^{1 / 2} g_{n} \\
\psi_{n}
\end{array}\right)
$$

Define a linear operator $\mathcal{Q}: H_{\partial}^{+} \times H_{1} \rightarrow H$ as shown below: for $(g, \psi)^{\tau} \in \mathcal{M}_{0}$, set

$$
\mathcal{Q}\left(\begin{array}{c}
g \\
\psi
\end{array}\right)=\sum_{n=k+1}^{\infty} \alpha_{n} x_{n}
$$

in the orthogonal complement of $\mathcal{M}_{0}$ in $H_{\partial}^{+} \times H_{1}$, define $\mathcal{Q}$ by the zero operator.

It is easily checked that (i) holds. For any $x \in D\left(A^{1 / 2}\right)$, by $(2.20)$ we have

$$
\mathcal{Q B} x=\sum_{n=k+1}^{\infty} \sqrt{\lambda_{n}} \alpha_{n} x_{n}=A^{1 / 2} x .
$$

From the proof of Theorem 2.2, we know that, if $\Gamma x=0, \forall x \in D\left(A^{1 / 2}\right)$, then $\mathcal{Q}$ can be defined from $H_{1}$ to $H$. In fact, we have the following result.

Theorem 2.3. Let $\left(B, H_{1}\right)$ be a pseudo-square-root of $A$. Set

$$
\mathbb{R}_{0}=\overline{\operatorname{span}}\left\{\psi_{n} \mid n \geq k+1\right\}
$$

Then $M_{0}=D(A)$ if and only if there exists a bounded linear operator $Q: H_{1} \rightarrow H$ such that

(i) $\|Q\| \leq 1, Q: \mathbb{R}_{0} \rightarrow H$ is isometric;

(ii) $A^{1 / 2} x=Q B x, \forall x \in D\left(A^{1 / 2}\right)$. 
Proof. The "only if" part follows from Theorem 2.2 .

The "if" part. By (ii) we have that $\sqrt{\lambda_{n}} x_{n}=A^{1 / 2} x_{n}=Q B x_{n}, \forall n \geq k+1$, i.e.,

$$
Q \psi_{n}=x_{n}, \quad n=k+1, k+2, \ldots
$$

The isometry of $Q: \mathbb{R}_{0} \rightarrow H$ yields

$$
\left(\psi_{n}, \psi_{m}\right)_{1}=\left(x_{n}, x_{m}\right), \quad \forall n, m \geq k+1
$$

From the orthogonality of $\left\{x_{n}\right\}$ in $H$ we obtain that

$$
\left[x_{n}, x_{m}\right]=\lambda_{m}\left[\left(x_{n}, x_{m}\right)-\sqrt{\frac{\lambda_{n}}{\lambda_{m}}}\left(\psi_{n}, \psi_{m}\right)_{1}\right]=0, \quad \forall n, m \geq k+1
$$

In addition, we have by a calculation that

$$
\left[x_{n}, x\right]=\overline{\left[x, x_{n}\right]}=0, \quad \forall x \in D(A), 1 \leq n \leq k .
$$

For any $x, y \in D(A), x=\sum_{n=1}^{\infty} \alpha_{n} x_{n}, y=\sum_{n=1}^{\infty} \beta_{n} x_{n}$, we have from (2.24) and (2.25) that

$$
[x, y]=\sum_{n=1}^{\infty} \sum_{m=1}^{\infty} \alpha_{n} \overline{\beta_{m}}\left[x_{n}, x_{m}\right]=0 .
$$

Thus $M_{0}=D(A)$.

Example 2.4. Let $A$ and $B$ be given by Example 2.3.

(i) Let $A$ have any one of the boundary conditions of (2.11), (3.12), and (2.13). Then $M_{0}=D(A)$. By Theorem 2.3, there is a bounded linear operator $Q: L^{2}(0,1) \rightarrow L^{2}(0,1)$ such that

$$
A^{1 / 2} u=Q\left(i u^{\prime}\right), \quad \forall u \in D\left(A^{1 / 2}\right)
$$

Set

$$
F(t, s)=\sum_{n=1}^{\infty}\left(Q x_{n}\right)(t) \overline{x_{n}(s)}, \quad 0 \leq t, s \leq 1
$$

Then

$$
A^{1 / 2} u=i \int_{0}^{1} F(t, \xi) u^{\prime}(\xi) d \xi, \quad \forall u \in D\left(A^{1 / 2}\right) .
$$

(ii) Let the boundary conditions of $A$ be given as $(2.14)$. Then $(\mathbb{C}, 1, \Gamma)$ is a positive boundary space of $A$ corresponding to $B$, where $\Gamma u=u(0), \forall u \in H^{1}(0,1)$. By Theorem 2.2 , there is a bounded linear operator $\mathcal{Q}: \mathbb{C} \times L^{2}(0,1) \rightarrow L^{2}(0,1)$ such that

$$
A^{1 / 2} u=\mathcal{Q}\left(\begin{array}{c}
u(0) \\
i u^{\prime}(\cdot)
\end{array}\right), \quad \forall u \in D\left(A^{1 / 2}\right)=\left\{u \mid u \in H^{1}(0,1), u(1)=0\right\}
$$

Let $\left\{x_{n}(\cdot)\right\}$ be the orthonormal basis of $L^{2}(0,1)$ consisting of the eigenfunctions of $A$. Set

$$
\mathcal{Y}_{n}(t)=\mathcal{Q}\left(\begin{array}{c}
x_{n}(0) \\
0
\end{array}\right) \in L^{2}(0,1), \quad n \geq 1,0 \leq t \leq 1
$$




$$
\begin{gathered}
\mathcal{Z}_{n}(t)=\mathcal{Q}\left(\begin{array}{c}
0 \\
x_{n}
\end{array}\right) \in L^{2}(0,1), \quad n \geq 1,0 \leq t \leq 1, \\
F_{\partial}(t, s)=\sum_{n=1}^{\infty} \mathcal{Y}_{n}(t) \overline{x_{n}(s)}, \quad F(t, s)=\sum_{n=1}^{\infty} \mathcal{Z}_{n}(t) \overline{x_{n}(s)}, \quad 0 \leq t, s \leq 1 .
\end{gathered}
$$

Then we have that

$$
\begin{aligned}
A^{1 / 2} u & =\mathcal{Q}\left(\begin{array}{c}
u(0) \\
0
\end{array}\right)+\mathcal{Q}\left(\begin{array}{c}
0 \\
i u^{\prime}(\cdot)
\end{array}\right) \\
& =\sum_{n=1}^{\infty}\left(u, x_{n}\right)_{L^{2}(0,1)} \mathcal{Y}_{n}(t)+\sum_{n=1}^{\infty}\left(i u^{\prime}, x_{n}\right)_{L^{2}(0,1)} \mathcal{Z}_{n}(t) \\
& =\int_{0}^{1} F_{\partial}(t, \xi) u(\xi) d \xi+i \int_{0}^{1} F(t, \xi) u^{\prime}(\xi) d \xi, \quad \forall u \in D\left(A^{1 / 2}\right)
\end{aligned}
$$

Finally, we consider the relationship between $A^{1 / 2}$ and $B$ from a different point of view. For the elastic "beam" operator $A$, i.e., $A u=u^{(4)}(\cdot), u \in D(A) \subset H^{4}(0,1)$, and the assumption that the boundary conditions of $A$ are "symmetric" (see Section 3, (3.3)), D. L. Russell [2] proved that there is a bounded linear operator $P: L^{2}(0,1) \rightarrow L^{2}(0,1)$ such that

$$
P A^{1 / 2} u=-u^{\prime \prime}(\cdot), \quad \forall u \in D\left(A^{1 / 2}\right) .
$$

Here we have the following general result.

TheOREm 2.4. Let $\left(B, H_{1}\right)$ be a pseudo-square-root of $A$. Then there is a bounded linear operator $P: H \rightarrow H_{1}$ with $\|P\| \leq 1$ such that

$$
P A^{1 / 2} x=B x, \quad \forall x \in D\left(A^{1 / 2}\right)
$$

Proof. Define $P: H \rightarrow H_{1}$ by

$$
P x=\sum_{n=k+1}^{\infty} \alpha_{n} \psi_{n}, \quad \forall x=\sum_{n=1}^{\infty} \alpha_{n} x_{n} \in H
$$

Let $\left(H_{\partial}^{+}, \Upsilon, \Gamma\right)$ be a positive boundary space of $A$ corresponding to $B$. Set

$$
\left(\begin{array}{c}
g \\
\psi
\end{array}\right)=\sum_{n=k+1}^{\infty} \alpha_{n}\left(\begin{array}{c}
\Upsilon^{1 / 2} g_{n} \\
\psi_{n}
\end{array}\right), \quad \forall x=\sum_{n=1}^{\infty} \alpha_{n} x_{n} \in H
$$

Since $\sum_{n=1}^{\infty}\left|\alpha_{n}\right|^{2}<+\infty$ we know that $(g, \psi)^{\tau} \in H_{\partial}^{+} \times H_{1}$. Hence

$$
P x=\psi \in H_{1}, \quad \forall x \in H
$$

and

$$
\|P x\|_{1}^{2} \leq\|g\|_{\partial_{+}}^{2}+\|\psi\|_{1}^{2}=\sum_{n=k+1}^{\infty}\left|\alpha_{n}\right|^{2} \leq\|x\|^{2}, \quad \forall x=\sum_{n=1}^{\infty} \alpha_{n} x_{n} \in H
$$


that is, $\|P\| \leq 1$. Furthermore, for any $x=\sum_{n=1}^{\infty} \alpha_{n} x_{n} \in D\left(A^{1 / 2}\right)$, we have

$$
P A^{1 / 2} x=\sum_{n=k+1}^{\infty} \sqrt{\lambda_{n}} \alpha_{n} P x_{n}=B x
$$

By Theorem 2.4, if $P: H \rightarrow \mathbb{R}_{0}$ has a bounded inverse $P^{-1}: \mathbb{R}_{0} \rightarrow H$, then $A^{1 / 2}$ can be written as $A^{1 / 2}=P^{-1} B$, where $P^{-1}: \mathbb{R}_{0} \rightarrow H$ is not isometric in general. In particular, if $\left\{\psi_{n} \mid n \geq k+1\right\}$ is an orthogonal set in $H_{1}$ (which is not true in general), then the above assertion holds. In the following we give a necessary condition on the orthogonality of $\left\{\psi_{n} \mid n \geq k+1\right\}$ if $\operatorname{dim}\left(H_{\partial}^{+}\right)<+\infty$ by which an error in [2] can be corrected (see Sec. 3).

Proposition 2.5. Let $\left(B, H_{1}\right)$ be a pseudo-square-root of $A$ and $\left(H_{\partial}^{+}, \Upsilon, \Gamma\right)$ a positive boundary space of $A$ corresponding to $B$ with $\operatorname{dim}\left(H_{\partial}^{+}\right)<\infty$. If $\left\{\psi_{n} \mid n \geq k+1\right\}$ is an orthogonal set in $H_{1}$, then there is a positive integer $n_{0}$ such that

$$
\left[x_{n}, x_{n}\right]=0, \quad \forall n \geq n_{0} .
$$

Proof. Suppose $\operatorname{dim}\left(H_{\partial}^{+}\right)=m$. By an argument similar to the proof of Proposition 2.2 , we have

$$
\left(x_{n}, x_{j}\right)=\sqrt{\frac{\lambda_{n}}{\lambda_{j}}}\left[\left(\Upsilon^{1 / 2} g_{n}, \Upsilon^{1 / 2} g_{j}\right)_{\partial}^{+}+\left(\psi_{n}, \psi_{j}\right)_{1}\right], \quad \forall n, j \geq k+1 .
$$

Thus $\left\{\Upsilon^{1 / 2} g_{n} \mid n \geq k+1\right\}$ is an orthogonal set in $H_{\partial}^{+}$. Since $\operatorname{dim}\left(H_{\partial}^{+}\right)=m$, there are at most $m$ nonzero elements in $\left\{\Upsilon^{1 / 2} g_{n} \mid n \geq k+1\right\}$. Therefore, there exists a positive integer $n_{0} \geq k+1$ such that

$$
\Upsilon^{1 / 2} g_{n}=0, \quad \forall n \geq n_{0}
$$

From (2.24), we have

$$
\left[x_{n}, x_{n}\right]=\lambda_{n}\left[\left(x_{n}, x_{n}\right)-\left(\psi_{n}, \psi_{n}\right)_{1}\right]=0, \quad \forall n \geq n_{0}
$$

3. Applications to elastic "beam" operators. Let $H=L^{2}(0,1)$. In this section, we shall consider elastic "beam" operators defined by

$$
A u=u^{(4)}(\cdot), \quad u \in D(A) \subset H^{4}(0,1) .
$$

Since

$$
(A u, u)_{L^{2}(0,1)}=\left.u^{\prime \prime \prime} \bar{u}\right|_{0} ^{1}-\left.u^{\prime \prime} \overline{u^{\prime}}\right|_{0} ^{1}+\int_{0}^{1}\left|u^{\prime \prime}\right|^{2} d t, \quad \forall u \in D(A),
$$

it is supposed that

$$
\left.u^{\prime \prime \prime} \bar{u}\right|_{0} ^{1}-\left.u^{\prime \prime} \overline{u^{\prime}}\right|_{0} ^{1} \geq 0, \quad \forall u \in D(A) .
$$

Set $H_{1}=L^{2}(0,1)$. Define $B: L^{2}(0,1) \rightarrow L^{2}(0,1)$ by

$$
D(B)=H^{2}(0,1), \quad B u=-D^{2} u=-u^{\prime \prime}(\cdot) .
$$

The following proposition can be easily verified. 
Proposition 3.1. Let $A$ be a nonnegative selfadjoint operator that satisfies (3.1) and (3.2). Then $\left(-D^{2}, L^{2}(0,1)\right)$ is a pseudo-square-root of $A$.

First, we introduce a main result given in [2]. Consider the real Hilbert space $L^{2}(0,1)$. Set

$$
\begin{gathered}
B(u, v)=u^{\prime \prime \prime}(\cdot) v(\cdot)-u^{\prime \prime}(\cdot) v^{\prime}+u^{\prime}(\cdot) v^{\prime \prime}(\cdot)-u(\cdot) v^{\prime \prime \prime}(\cdot), \quad \forall u, v \in D(A) \\
\psi_{n}(\cdot)=-\left(\sqrt{\lambda_{n}}\right)^{-1} D^{2} x_{n}(\cdot), \quad n \geq k+1
\end{gathered}
$$

where $\lambda_{n}, x_{n}$, and $k$ are the same as those in Sec. 2. D. L. Russell in [2] has shown the following result.

Let $A$ be a nonnegative selfadjoint operator on $L^{2}(0,1)$ with the compact resolvent such that (3.1) and (3.2) hold. Suppose that

$$
B(u, v)=0, \quad \forall u, v \in D(A)
$$

at $x=0$ and at $x=1$. Then

(i) there is a bounded linear operator $P: L^{2}(0,1) \rightarrow L^{2}(0,1)$ such that

$$
P A^{1 / 2} u=-D^{2} u, \quad \forall u \in D\left(A^{1 / 2}\right) .
$$

(ii) Set $\mathbb{R}_{0}=\overline{\operatorname{span}}\left\{\psi_{n} \mid n \geq k+1\right\}$. Then $P$ has a bounded inverse on $\mathbb{R}_{0}$, which extends to a bounded linear operator $Q$ on $L^{2}(0,1)$, so that likewise

$$
A^{1 / 2} u=Q\left(-D^{2}\right) u, \quad \forall u \in D\left(A^{1 / 2}\right) .
$$

From the proof of the above assertion (ii) in [2, pp. 761-765] we know that the assertion (ii) is based on the assertion that $\left\{\psi_{n} \mid n \geq k+1\right\}$ is an orthogonal set in $L^{2}(0,1)$. Unfortunately, Proposition 3.2 below shows that in general $\left\{\psi_{n} \mid n \geq k+1\right\}$ is not an orthogonal set in $L^{2}(0,1)$ under the assumption (3.3) only. Therefore, the proof of the assertion (ii) given in [2] failed. By Theorem 2.2 and Theorem 2.3, the assertion (ii) can be revised (see Example 3.1 below).

EXAMPLE 3.1. Let us consider a cantilever with elastic forces applying at the free end, $x=1$. The boundary conditions become

$$
u(0)=u^{\prime}(0)=0, \quad u^{\prime \prime \prime}(1)-\alpha u(1)=0, \quad u^{\prime \prime}(1)+\beta u^{\prime}(1)=0
$$

where $\alpha>0, \beta>0$.

Set $H_{\partial}^{+}=\mathbb{C}^{2}$. Define $\Upsilon: \mathbb{C}^{2} \rightarrow \mathbb{C}^{2}$ by

$$
\Upsilon=\left(\begin{array}{ll}
\alpha & 0 \\
0 & \beta
\end{array}\right)
$$

Define $\Gamma: H^{2}(0,1) \rightarrow \mathbb{C}^{2}$ by

$$
\Gamma u=\left(\begin{array}{c}
u(1) \\
u^{\prime}(1)
\end{array}\right), \quad \forall u \in H^{2}(0,1) .
$$


It can easily be verified that

$$
[u, v]=\alpha u(1) \overline{v(1)}+\beta u^{\prime}(1) \overline{v^{\prime}(1)}, \quad \forall u, v \in D(A) .
$$

Then $\left(\mathbb{C}^{2}, \Upsilon, \Gamma\right)$ is a positive boundary space of $A$ corresponding to $-D^{2}$. Since

$$
\begin{aligned}
{[u, v] } & =\alpha u(1) \overline{v(1)}+\beta u^{\prime}(1) \overline{v^{\prime}(1)}-u(0) \overline{v^{\prime \prime \prime}(0)}+u^{\prime}(0) \overline{v^{\prime \prime}(0)} \\
& =(\Upsilon \Gamma u, \Gamma v)_{\mathbb{C}^{2}}-u(0) \overline{v^{\prime \prime \prime}(0)}+u^{\prime}(0) \overline{v^{\prime \prime}(0)}, \quad \forall u \in H^{2}(0,1), v \in D(A),
\end{aligned}
$$

from Theorem 2.1 , we have

$$
\begin{aligned}
D\left(A^{1 / 2}\right) & =\left\{u \mid u \in H^{2}(0,1),[u, v]=(\Upsilon \Gamma u, \Gamma v)_{\mathbb{C}^{2}}, \forall v \in D(A)\right\} \\
& =\left\{u \mid u \in H^{2}(0,1), u(0)=u^{\prime}(0)=0\right\} .
\end{aligned}
$$

By Theorem 2.2, there is a bounded linear operator $\mathcal{Q}: \mathbb{C}^{2} \times L^{2}(0,1) \rightarrow L^{2}(0,1)$ such that

$$
A^{1 / 2} u=\mathcal{Q}\left(\begin{array}{c}
\sqrt{\alpha} u(1) \\
\sqrt{\beta} u^{\prime}(1) \\
-u^{\prime \prime}(\cdot)
\end{array}\right), \quad \forall u \in D\left(A^{1 / 2}\right) .
$$

By an argument similar to Example 2.4, we can find $F_{\partial_{1}}(t, \xi), F_{\partial_{2}}(t, \xi)$, and $F(t, \xi)$, for $0 \leq t, \xi \leq 1$, such that

$$
\begin{gathered}
A^{1 / 2} u=\sqrt{\alpha} \int_{0}^{1} F_{\partial_{1}}(t, \xi) u(\xi) d \xi+\sqrt{\beta} \int_{0}^{1} F_{\partial_{2}}(t, \xi) u^{\prime}(\xi) d \xi-\int_{0}^{t} F(t, \xi) u^{\prime \prime}(\xi) d \xi, \\
\forall u \in D\left(A^{1 / 2}\right) .
\end{gathered}
$$

Proposition 3.2. Let $A$ be the "beam" operator on $L^{2}(0,1)$ with the boundary conditions given by (3.5). Then (3.3) holds but $\left\{\psi_{n} \mid n \geq k+1\right\}$ is not an orthogonal set in $L^{2}(0,1)$, where $\psi_{n}=-\left(\sqrt{\lambda_{n}}\right)^{-1} x_{n}^{\prime \prime}(\cdot), \lambda_{n}$ is the eigenvalue of $A$, and $x_{n}(\cdot)$ is the eigenfunction of $A$ corresponding to $\lambda_{n}$, for all $n \geq 1$.

Proof. It is easily checked that (3.3) holds.

Suppose that $\left\{\psi_{n} \mid n \geq k+1\right\}$ is an orthogonal set in $L^{2}(0,1)$. By Proposition 2.5, there is a positive integer $n_{0}$ such that

$$
\left[x_{n}, x_{n}\right]=\alpha\left|x_{n}(1)\right|^{2}+\beta\left|x_{n}^{\prime}(1)\right|^{2}=0, \quad \forall n \geq n_{0} .
$$

Therefore, $x_{n}$ is a solution of the following boundary value problem:

$$
\left\{\begin{array}{l}
x_{n}^{(4)}(t)=\lambda_{n} x_{n}(t), \quad 0<t<1, \\
x_{n}(0)=x_{n}^{\prime}(0)=x_{n}(1)=x_{n}^{\prime}(1)=x_{n}^{\prime \prime}(1)=x_{n}^{\prime \prime \prime}(1)=0,
\end{array}\right.
$$

for all $n \geq n_{0}$. It is obvious that the problem (3.7) has the unique zero solution, for all $n \geq n_{0}$. Thus

$$
x_{n}=0, \quad \forall n \geq n_{0} .
$$

This is a contradiction.

REMARK 3.1. It should be noted that there is a modified inner product, as shown in Proposition 2.2, relative to which $\psi_{n}$ may be considered orthogonal. Thus, the statement made in [2] is correct if restricted to "SDE" boundary conditions (strictly distributed energy [2]). Without the SDE assumption, the potential energy form associated with the operator $A$ includes some boundary terms.

Finally, we conclude this section by giving an example derived from the pointwise control of a flexible manipulator arm [6]. 
EXAMPLE 3.2. Let $H=\mathbb{C}^{3} \times L^{2}(0,1)$ with the inner product

$$
\left(\Phi_{1}, \Phi_{2}\right)=\alpha_{1} \overline{\alpha_{2}}+\mu_{1} \overline{\mu_{2}}+\xi_{1} \overline{\xi_{2}}+\int_{0}^{1} \varphi_{1} \overline{\varphi_{2}} d t
$$

where $\Phi_{j}=\left(\alpha_{j}, \mu_{j}, \xi_{j}, \varphi_{j}\right)^{\tau} \in \mathbb{C}^{3} \times L^{2}(0,1)$. Define the linear operator $A$ by

$$
\begin{gathered}
A \tilde{\varphi}=\left(\begin{array}{c}
-\varphi^{\prime \prime}(0) \\
-\varphi^{\prime \prime \prime}(1) \\
\varphi^{\prime \prime}(1) \\
\varphi^{(4)}(\cdot)
\end{array}\right), \quad \tilde{\varphi}=\left(\begin{array}{c}
\varphi^{\prime}(0) \\
\varphi(1) \\
\varphi^{\prime}(1) \\
\varphi(\cdot)
\end{array}\right), \\
D(A)=\left\{\tilde{\varphi}=\left(\varphi^{\prime}(0), \varphi(1), \varphi^{\prime}(1), \varphi(\cdot)\right)^{\tau} \mid \varphi \in H^{4}(0,1), \varphi(0)=0\right\} .
\end{gathered}
$$

It is easily checked that $A$ is a nonnegative selfadjoint operator on $\mathbb{C}^{3} \times L^{2}(0,1)$.

Set $H_{1}=L^{2}(0,1)$. Define $B: \mathbb{C}^{3} \times L^{2}(0,1) \rightarrow L^{2}(0,1)$ by

$$
\begin{gathered}
B \tilde{\varphi}=-D^{2} \varphi=-\varphi^{\prime \prime}(\cdot), \quad \tilde{\varphi}=\left(\begin{array}{c}
\varphi^{\prime}(0) \\
\varphi(1) \\
\varphi^{\prime}(1) \\
\varphi(\cdot)
\end{array}\right), \\
D(B)=\left\{\tilde{\varphi}=\left(\varphi^{\prime}(0), \varphi(1), \varphi^{\prime}(1), \varphi(\cdot)\right)^{\tau} \mid \varphi \in H^{2}(0,1)\right\} .
\end{gathered}
$$

It is easily checked that $\left(B, L^{2}(0,1)\right)$ is a pseudo-square-root of $A$. Since

$$
[\tilde{\psi}, \tilde{\varphi}]=0, \quad \forall \tilde{\psi}, \tilde{\varphi} \in D(A)
$$

then $M_{0}=D(A)$.Since

$$
[\tilde{\psi}, \tilde{\varphi}]=-\psi(0) \overline{\varphi^{\prime \prime \prime}(0)}, \quad \forall \tilde{\psi} \in D(B), \tilde{\varphi} \in D(A),
$$

by Corollary 2.1, we have

$$
\begin{aligned}
D\left(A^{1 / 2}\right) & =\{\tilde{\psi} \mid \tilde{\psi} \in D(B),[\tilde{\psi}, \tilde{\varphi}]=0, \forall \tilde{\varphi} \in D(A)\} \\
& =\left\{\tilde{\psi}=\left(\psi^{\prime}(0), \psi(1), \psi^{\prime}(1), \psi(\cdot)\right)^{\tau} \mid \psi \in H^{2}(0,1), \psi(0)=0\right\} .
\end{aligned}
$$

Furthermore, by Theorem 2.3, there is a bounded linear operator $Q: L^{2}(0,1) \rightarrow \mathbb{C}^{3} \times$ $L^{2}(0,1)$ such that

$$
A^{1 / 2} \tilde{\psi}=-Q\left(\psi^{\prime \prime}\right), \quad \forall \tilde{\psi}=\left(\psi^{\prime}(0), \psi(1), \psi^{\prime}(1), \psi(\cdot)\right)^{\tau} \in D\left(A^{1 / 2}\right) .
$$

Since $Q: L^{2}(0,1) \rightarrow \mathbb{C}^{3} \times L^{2}(0,1)$ is bounded, $Q$ has the form

$$
Q u=\left(\begin{array}{c}
g_{1}(u) \\
g_{2}(u) \\
g_{3}(u) \\
Q_{0} u
\end{array}\right), \quad \forall u \in L^{2}(0,1)
$$

where $g_{j}(\cdot)$ are bounded linear functionals, for $j=1,2,3$, and $Q_{0}: L^{2}(0,1) \rightarrow L^{2}(0,1)$ is a bounded linear operator. By the Riesz theorem and an argument similar to Example 2.4, we obtain $f_{j} \in L^{2}(0,1)$ and $F(t, s), j=1,2,3,0 \leq t, s \leq 1$, such that

$$
A^{1 / 2} \tilde{\varphi}=\left(\begin{array}{c}
-\int_{0}^{1} f_{1}(\xi) \varphi^{\prime \prime}(\xi) d \xi \\
-\int_{0}^{1} f_{2}(\xi) \varphi^{\prime \prime}(\xi) d \xi \\
-\int_{0}^{1} f_{3}(\xi) \varphi^{\prime \prime}(\xi) d \xi \\
-\int_{0}^{1} F(\cdot, \xi) \varphi^{\prime \prime}(\xi) d \xi
\end{array}\right), \quad \forall \tilde{\varphi}=\left(\varphi^{\prime}(0), \varphi(1), \varphi^{\prime}(1), \varphi(\cdot)\right)^{\tau} \in D\left(A^{1 / 2}\right)
$$


4. Applications to $n$-dimensional "wave" operators. In this section we shall apply the results given in previous sections to a variety of boundary value problems of $n$-dimensional "wave" operators. To our knowledge, there presently is no available method for readily calculating the square root of high-dimensional Laplace operators. Let $\Omega \subset \mathbb{R}^{n}$ be a bounded domain of $\mathbb{R}^{n}$ with smooth boundary $\partial \Omega$ of class $C^{2}$ and $\frac{\partial}{\partial n}$ the normal derivative.

EXAmple 4.1. Let $\partial \Omega=\Gamma_{1} \cup \Gamma_{2} \cup \Gamma_{3}$. Consider the Laplace operator on $L^{2}(\Omega)$

$$
\begin{gathered}
D(A)=\left\{u\left|u \in H^{2}(\Omega), u\right|_{\Gamma_{1}}=0,\left.\frac{\partial u}{\partial n}\right|_{\Gamma_{2}}=0,\left.\left(\frac{\partial u}{\partial n}+\alpha u\right)\right|_{\Gamma_{3}}=0\right\} \\
A u=-\Delta u
\end{gathered}
$$

where $\alpha>0$.

Set $H_{1}=\left(L^{2}(\Omega)\right)^{n}$. Define the closed linear operator $B: L^{2}(\Omega) \rightarrow\left(L^{2}(\Omega)\right)^{n}$ by

$$
D(B)=H^{1}(\Omega), \quad B u=\nabla u=\left(\frac{\partial u}{\partial x_{1}}, \frac{\partial u}{\partial x_{2}}, \ldots, \frac{\partial u}{\partial x_{n}}\right)^{\tau} .
$$

It is easily checked that $\left(\nabla,\left(L^{2}(\Omega)\right)^{n}\right)$ is a pseudo-square-root of $A$. By Green's formula, we have

$$
\begin{aligned}
{[u, v] } & =-\int_{\partial \Omega} u \frac{\partial \bar{v}}{\partial n} d \sigma \\
& =-\int_{\Gamma_{1}} u \frac{\partial \bar{v}}{\partial n} d \sigma+\alpha \int_{\Gamma_{3}} u \bar{v} d \sigma, \quad \forall u \in H^{1}(\Omega), \forall v \in D(A)
\end{aligned}
$$

Set $H_{\partial}^{+}=L^{2}\left(\Gamma_{3}\right)$ and $\Upsilon=\alpha I$, where $I$ is the identity mapping on $L^{2}\left(\Gamma_{3}\right)$. Define $\Gamma: H^{1}(\Omega) \rightarrow L^{2}\left(\Gamma_{3}\right)$ by

$$
\Gamma u=\left.u\right|_{\Gamma_{3}}, \quad \forall u \in H^{1}(\Omega) .
$$

By (4.2), we have

$$
[u, v]=\alpha \int_{\Gamma_{3}} u \bar{v} d \sigma=(\Upsilon \Gamma u, \Gamma v)_{L^{2}\left(\Gamma_{3}\right)}, \quad \forall u, v \in D(A)
$$

Thus $\left(L^{2}\left(\Gamma_{3}\right), \alpha I, \Gamma\right)$ is a positive boundary space of $A$ corresponding to $\nabla$. From Theorem 2.1 and (4.2), we have

$$
D\left(A^{1 / 2}\right)=\left\{u\left|u \in H^{1}(\Omega), u\right|_{\Gamma_{1}}=0\right\} .
$$

By Theorem 2.2, there is a bounded linear operator $\mathcal{Q}: L^{2}\left(\Gamma_{3}\right) \times\left(L^{2}(\Omega)\right)^{n} \rightarrow L^{2}(\Omega)$ such that

$$
A^{1 / 2} u=\mathcal{Q}\left(\begin{array}{c}
\left.\sqrt{\alpha} u\right|_{\Gamma_{3}} \\
\nabla u
\end{array}\right), \quad \forall u \in D\left(A^{1 / 2}\right) .
$$

Suppose that $\left\{x_{j}(\zeta)\right\}$ is the orthonormal basis of $L^{2}(\Omega)$ consisting of the eigenfunctions of $A$ and $e_{j}=(0, \ldots, 1, \ldots, 0)^{\tau} \in \mathbb{R}^{n+1}, j=0,1, \ldots, n$. Setting

$y_{j}(\zeta)=\mathcal{Q}\left(\left.x_{j}\right|_{\Gamma_{3}} e_{0}\right) \in L^{2}(\Omega), \quad Z_{j, m}(\zeta)=\mathcal{Q}\left(x_{j} e_{m}\right) \in L^{2}(\Omega), \quad j \geq 1,1 \leq m \leq n, \zeta \in \Omega$, 


$$
F_{\partial}(\zeta, \xi)=\sum_{j=1}^{\infty} y_{j}(\zeta) \overline{x_{j}(\xi)}, \quad F_{m}(\zeta, \xi)=\sum_{j=1}^{\infty} Z_{j, m}(\zeta) x_{j}(\xi), \quad \forall \zeta, \xi \in \Omega, 1 \leq m \leq n
$$

We finally obtain

$$
A^{1 / 2} u=\sqrt{\alpha} \int_{\Omega} F_{\partial}(\zeta, \xi) u(\xi) d \xi+\sum_{m=1}^{n} \int_{\Omega} F_{m}(\zeta, \xi) \frac{\partial}{\partial x_{m}} u(\xi) d \xi, \quad \forall u \in D\left(A^{1 / 2}\right) .
$$

EXAmPLE 4.2. Let $A$ be the Laplace operator with the domain

$$
D(A)=\left\{u \mid u \in H^{2}(\Omega), \int_{\partial \Omega} u d \sigma=0, \frac{\partial u}{\partial n}=\frac{1}{|\partial \Omega|} \int_{\partial \Omega} \Delta u d \sigma\right\},
$$

where $|\partial \Omega|$ is the measure of $\partial \Omega$ in $\mathbb{R}^{n-1}$.

Let $\left(\nabla,\left(L^{2}(\Omega)\right)^{n}\right)$ be given by Example 4.1. Then $\left(\nabla,\left(L^{2}(\Omega)\right)^{n}\right)$ is a pseudo-squareroot of $A$. Since

$$
[u, v]=-\int_{\partial} u \frac{\partial \bar{v}}{\partial n} d \sigma=\frac{-1}{|\partial \Omega|} \int_{\partial \Omega} \Delta \bar{v} d \sigma \int_{\partial} u d \sigma=0, \quad \forall u, v \in D(A),
$$

then $M_{0}=D(A)$. By Corollary 2.1, we have that

$$
\begin{aligned}
D\left(A^{1 / 2}\right) & =\left\{u \mid u \in H^{1}(\Omega),[u, v]=0, \forall v \in D(A)\right\} \\
& =\left\{u \mid u \in H^{1}(\Omega), \int_{\partial \Omega} u d \sigma=0\right\} .
\end{aligned}
$$

By Theorem 2.3, there exists a bounded linear operator $Q:\left(L^{2}(\Omega)\right)^{n} \rightarrow L^{2}(\Omega)$ such that

$$
A^{1 / 2} u=Q(\nabla u), \quad u \in D\left(A^{1 / 2}\right) .
$$

By an argument similar to Example 4.1 , there exist $F_{m}(\zeta, \xi)$, for $m=1,2, \ldots, n$ such that

$$
A^{1 / 2} u=\sum_{m=1}^{n} \int_{\Omega} F_{m}(\zeta, \xi) \frac{\partial u(\xi)}{\partial x_{m}} d \xi, \quad \forall u \in D\left(A^{1 / 2}\right)
$$

ExAmple 4.3. Let $A$ be the Laplace operator with the domain

$$
D(A)=\left\{u\left|u \in H^{2}(\Omega), \int_{\partial \Omega} \frac{\partial u}{\partial n} d \sigma=0, u\right|_{\partial \Omega}=\text { constant }\right\} .
$$

Then $\left(\nabla,\left(L^{2}(\Omega)\right)^{n}\right)$ is a pseudo-square-root of $A$.

Since

$$
[u, v]=-\int_{\partial \Omega} u \frac{\partial \bar{v}}{\partial n} d \sigma=-\left.u\right|_{\partial \Omega} \int_{\partial \Omega} \frac{\partial \bar{v}}{\partial n} d \sigma=0, \quad \forall u, v \in D(A),
$$

we know that $M_{0}=D(A)$. Suppose $u \in H^{1}(\Omega)$ such that

$$
[u, v]=-\int_{\partial \Omega} u \frac{\partial \bar{v}}{\partial n} d \sigma=0, \quad \forall v \in D(A) .
$$

It is easily checked by the trace operator theorem that

$$
\int_{\partial \Omega} u g d \sigma=0, \quad \forall g \in L^{2}(\partial \Omega), \quad \int_{\partial \Omega} g d \sigma=0 .
$$

Thus $\left.u\right|_{\partial \Omega}=$ constant. Therefore, by Corollary 2.1, we have

$$
\begin{aligned}
D\left(A^{1 / 2}\right) & =\left\{u \mid u \in H^{1}(\Omega),[u, v]=0, \forall v \in D(A)\right\} \\
& =\left\{u\left|u \in H^{1}(\Omega), u\right|_{\partial \Omega}=\text { constant }\right\} .
\end{aligned}
$$

In addition, by Theorem $2.3, A^{1 / 2}$ has the form of (4.6). 
EXAMPLE 4.4. Let

$$
a(\zeta)=\left(a_{i j}(\zeta)\right)_{n \times n}, \quad a_{i j} \in C^{1}(\bar{\Omega}), 1 \leq i, j \leq n,
$$

be a symmetric matrix on $\mathbb{C}^{n}$ and suppose that there is $\delta>0$ such that

$$
\sum_{j=1}^{n} \sum_{i=1}^{n} a_{i j}(\zeta) \mu_{i} \overline{\mu_{j}} \geq \delta|\mu|^{2}, \quad \forall \mu=\left(\mu_{1}, \mu_{2}, \ldots, \mu_{n}\right)^{\tau} \in \mathbb{C}^{n}, \zeta \in \bar{\Omega} .
$$

Define $A$ by

$$
\begin{aligned}
& D(A)=\left\{u\left|u \in H^{2}(\Omega), u\right|_{\partial \Omega}=0\right\}, \\
& A u=-\sum_{j=1}^{n} \frac{\partial}{\partial x_{j}}\left(\sum_{i=1}^{n} a_{i j}(\zeta) \frac{\partial u}{\partial x_{i}}\right) .
\end{aligned}
$$

It is easily checked that $A$ is a nonnegative selfadjoint operator on $L^{2}(\Omega)$.

Set $H_{1}=\left(L^{2}(\Omega)\right)^{n}$. Define $B: L^{2}(\Omega) \rightarrow\left(L^{2}(\Omega)\right)^{n}$ by

$$
D(B)=H^{1}(\Omega), \quad B u=(a(\zeta))^{1 / 2} \nabla u .
$$

It is easily checked that $\left((a(\zeta))^{1 / 2} \nabla,\left(L^{2}(\Omega)\right)^{n}\right)$ is a pseudo-square-root of $A$. By Green's formula, we have

$$
[u, v]=-\int_{\partial \Omega} u \sum_{j=1}^{n}\left(\sum_{i=1}^{n} \overline{a_{i j}} \frac{\partial \bar{v}}{\partial x_{i}}\right) n_{j} d \sigma=0, \quad \forall u, v \in D(A),
$$

where $n=\left(n_{1}, n_{2}, \ldots, n_{n}\right)$ is the unit normal direction of $\partial \Omega$. Thus $M_{0}=D(A)$. Hence

$$
\begin{aligned}
D\left(A^{1 / 2}\right) & =\left\{u \mid u \in H^{1}(\Omega),[u, v]=0, \forall v \in D(A)\right\} \\
& =\left\{u\left|u \in H^{1}(\Omega), u\right|_{\partial \Omega}=0\right\} .
\end{aligned}
$$

By Theorem 2.3, there is a bounded linear operator $Q:\left(L^{2}(\Omega)\right)^{n} \rightarrow L^{2}(\Omega)$ such that

$$
A^{1 / 2} u=Q\left((a(\zeta))^{1 / 2} \nabla u\right), \quad \forall u \in D\left(A^{1 / 2}\right) .
$$

By an argument similar to Example $4.2, A^{1 / 2}$ also has a form of (4.6).

\section{REFERENCES}

[1] G. Chen and D. L. Russell, A mathematical model for linear elastic systems with structural damping, Quart. Appl. Math. 39, 433-454 (1982)

[2] D. L. Russell, On the positive square root of the fourth derivative operator, Quart. Appl. Math. XLVI, 751-773 (1988)

[3] A. V. Balakrishnan, Damping operators in continuum models of flexible structures: Explicit models for proportional damping in beam torsion, J. Differential Integral Equations 3, no. 2, 381-396 (1990). MR 91b:73063

[4] A. V. Balakrishnan, Damping operators in continuum models of flexible structure: Explicit models for proportional damping in beam bending with end-bodies, Appl. Math. Optim. 21, 315-334 (1990). MR 91i: 73035

[5] A. V. Balakrishnan, Fractional powers of closed operators and the semigroups generated by them, Pacific J. Math. 10, 417-437 (1960). MR 22:5899

[6] A. G. Chassiakos and G. A. Berkey, Pointwise control of a flexible manipulator arm, Proceedings of IFAC Symposium on Robot Control, Barcelona, Spain, November 1985, pp. 181-185 